\title{
Role of spin-orbit interaction in the ultrafast demagnetization of small iron clusters
}

\author{
Maria Stamenova, ${ }^{*}$ Jacopo Simoni, and Stefano Sanvito \\ School of Physics, AMBER and CRANN Institute, Trinity College, Dublin 2, Ireland \\ (Received 4 November 2015; revised manuscript received 12 May 2016; published 19 July 2016)
}

\begin{abstract}
The ultrafast demagnetization of small iron clusters initiated by an intense optical excitation is studied from the time-dependent spin density functional theory (TDSDFT). In particular we investigate the effect of the spin-orbit interaction on the onset of the demagnetization process. It is found that demagnetization occurs locally, in the vicinity of the atomic sites, and the initial rate of spin loss, coherent with the laser field, is proportional to the square of the ionic spin-orbit coupling strength $\lambda$. A simplified quantum spin model comprising spin-orbit interaction and a time-dependent magnetic field is found to be the minimal model able to reproduce our $a b$ initio results. The model predicts the $\lambda^{2}$ dependence of the onset rate of demagnetization when it is solved either analytically for the small $t$ regime, or numerically integrated in the time domain. Our findings are supported by additional TDSDFT simulations of clusters made of Co and $\mathrm{Ni}$.
\end{abstract}

DOI: 10.1103/PhysRevB.94.014423

\section{INTRODUCTION}

Achieving control over the magnetization dynamics at the femtosecond timescale is a desirable asset for new magnetic data storage technologies. The ultrafast optical demagnetization (UOD) phenomenon, discovered by Beaurepaire et al. [1], in which an intense femtosecond laser pulse induces an abrupt and dramatic loss of magnetization in a metallic film, initiated what is now the highly active field of femtomagnetism. Typical UOD experiments are based on the pump-probe method, where a femtosecond laser pulse in the optical range (pump) is beamed onto the magnetic sample and then a delayed short electromagnetic pulse (probe) is used to detect the magnetic response through possible linear or nonlinear magneto-optical effects [2]. By varying the time delay between the pump and the probe the magnetization dynamics can be reconstructed in the time domain over a typical range spanning from a few femtoseconds to a few picoseconds. The rapid demagnetization process that develops over this time can be characterized by two distinct stages: (i) a coherent stage in the first few tens of fs when the light interacts with the electrons and (ii) a relaxation stage when hot electrons and spins interact with each other and with the lattice so as to thermalize. Although the role of the particular microscopic spin-flip mechanisms is often unclear and dependent on the details of the magnet investigated, the thermalization process is in general tractable through empirical three-temperature models $[1,3]$, which establish rate equations between the spin, electron, and phonon systems. In contrast, theory for the coherent stage is rather unsettled and spans a range of different views (not necessarily mutually exclusive), from relativistic accounts of the direct photon-spin coupling [4] to semiclassical transport models [5].

Experimental works, focused on the coherent regime, have described a strong dependence of the rate of UOD on the material and, particularly, its spin-orbit coupling (SOC) properties. For instance, it has been reported [6] that materials exhibiting stronger SOC demagnetize significantly faster than

*stamenom@tcd.ie lighter ones. The SOC has been identified as a key component enabling UOD also in earlier theoretical works based on model Hamiltonian [7]. Very recently time-dependent spin-density functional theory (TDSDFT) calculations [8] have provided another confirmation of its essential role for the ultrafast laser-induced loss of spin in bulk transition metals. In this paper we seek to gain further understanding of the microscopic mechanisms responsible for the very initiation of the UOD. We employ the most practically-applicable first-principles theoretical framework, the TD(S)DFT $[9,10]$, which allows us to simulate the UOD process for real multiatomic clusters directly in the time domain and for experimentally-relevant times. We demonstrate that the very onset of the demagnetization is triggered by the electronic charge response to the electric field of the pulse. The charge and spin currents generated give rise to an effective magnetic field which, in combination with the SOC, facilitates spin flips and a global spin decay. We also establish that the initial, coherent demagnetization rate is proportional to the square of the ionic SOC strength for a number of similar clusters made of other transition-metal atoms.

In particular, we focus on an iron cluster- $-\mathrm{Fe}_{6}$. Its ground state (GS) geometry, as predicted by LSDA calculations [11,12], is a compressed $D_{2 h}$ distorted octahedron [see inset of Fig. 1(a)] with bond lengths in the range 2.29-2.53 $\AA$, and this is the geometry we adhere to. The corresponding GS spin of $\mathrm{Fe}_{6}$ in LSDA is $2 S=20 \hbar$. It is well known that open $d$-shell systems are problematic to local approximations of the exchange and correlation (XC) functional. Here, however, our intention is to study the generality of the spin dynamics so that possible quantitative features are not important at this time. We consider $\mathrm{Fe}_{6}$ in its GS geometry as in Ref. [12] [see Fig. 1(a)] and adopt the adiabatic temporal extension [13] of the local spin-density approximation (ALSDA), parametrized by Perdew and Wang [14] and implemented in the Octopus code [15].

The paper is organized as follows. In Sec. II we discuss the equations of motion for the spin density, which stem from the TDSDFT implementation that we use. Section III contains our main TDDFT results, which are then interpreted and understood through a minimal spin model introduced 

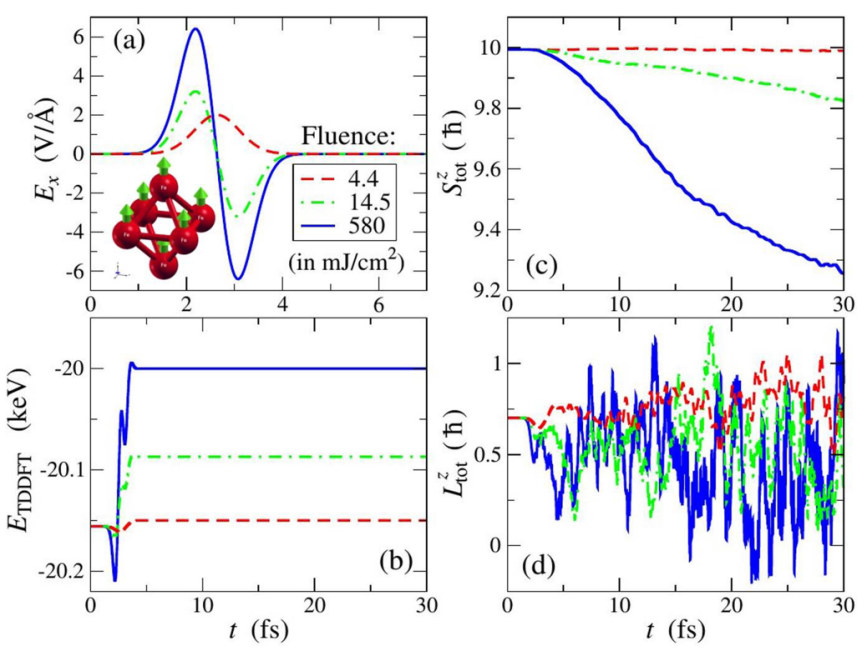

FIG. 1. (a) Typical electric field pulses used to excite the $\mathrm{Fe}_{6}$ cluster (cartooned as inset) and their corresponding fluences $\frac{c \varepsilon_{0}}{2} \int E_{x}^{2}(t) d t$. Time evolution of the total TDSDFT energy (b), total spin (c), and total KS angular momentum (d) of the cluster when subjected to each of the pulses in panel (a) with corresponding color code on all panels.

in Sec. IV. Finally, in Sec. V we quantify and compare the demagnetization of similar six-atom clusters of the same geometry but different materials ( $\mathrm{Ni}$ and $\mathrm{Co}$ ) as a function of their DFT-computed effective atomic SOC. We then conclude in Sec. VI. The paper is also completed by an Appendix, where we discuss in some detail aspects related to the angular momentum conservation.

\section{EQUATIONS OF MOTION FOR SPIN}

In all our calculations spin dynamics is initiated by a single intense electric field pulse (we neglect the magnetic field component). We solve the time-dependent Kohn-Sham (KS) equations

$$
i \hbar \frac{\partial}{\partial t} \psi_{j}(\vec{r}, t)=H_{\mathrm{KS}}(\vec{r}, t) \psi_{j}(\vec{r}, t),
$$

where $H_{\mathrm{KS}}(\vec{r}, t)$ is a $2 \times 2$ matrix in spin space to account for noncollinearity, $\psi_{j}$ are two-dimensional spinors, and we consider the time-dependent KS Hamiltonian with electric field $\vec{E}(t)$ introduced in the length gauge

$$
\begin{aligned}
H_{\mathrm{KS}}(\vec{r}, t)= & -\frac{\hbar^{2} \nabla^{2}}{2 m}+v_{\mathrm{s}}(\vec{r}, t)-\frac{2 \mu_{\mathrm{B}}}{\hbar} \hat{\vec{S}} \cdot \vec{B}_{\mathrm{xc}}(\vec{r}, t) \\
v_{\mathrm{s}}(\vec{r}, t)= & \sum_{\vec{R}_{I}} V_{\mathrm{PP}}\left(\left|\vec{r}-\vec{R}_{I}\right|\right)+\int d^{3} r^{\prime} \frac{n\left(\vec{r}^{\prime}\right)}{\left|\vec{r}-\vec{r}^{\prime}\right|} \\
& +v_{\mathrm{xc}}(\vec{r}, t)+\vec{r} \cdot \vec{E}(t) .
\end{aligned}
$$

We substitute the ionic potentials, centered at each site $\vec{R}_{I}$, with soft norm-conserving fully relativistic pseudopotentials that reproduce correctly the semicore and valence wave functions beyond a certain core radius $[16-18]^{1}$

$$
\begin{aligned}
V_{\mathrm{PP}}(r)= & \sum_{l} \sum_{m=-l}^{l}\left(\bar{V}_{l}^{\mathrm{ion}}(r)+\frac{1}{4} V_{l}^{\mathrm{SO}}(r)+\alpha V_{l}^{\mathrm{SO}}(r) \hat{\vec{L}} \cdot \hat{\vec{S}}\right) \\
& \times|l, m\rangle\langle l, m| .
\end{aligned}
$$

Here, $\hat{\vec{S}}$ is the spin operator, $\hat{\vec{L}}_{I}$ is the angular momentum operator associated to the atomic center " $I$ ", while the scalar part of the pseudopotential $\bar{V}_{l}^{\text {ion }}(r)$ includes the effect of the mass shift and the Darwin term. $V_{l}^{\mathrm{SO}}(r)$ defines the range of the $\mathrm{SOC}$ term, ${ }^{2}$ and $\alpha$ is just an added tunable parameter which is equal to 1 in the correct formulation. Within the ALSDA, at every time $t$ the XC potentials are calculated as

$$
v_{\mathrm{xc}}(\vec{r}, t)=\left.\frac{\delta E_{\mathrm{xc}}^{\mathrm{LDA}}}{\delta n}\right|_{\substack{n(\vec{r}, t) \\ \vec{s}(\vec{r}, t)}}, \vec{B}_{\mathrm{xc}}(\vec{r}, t)=\left.\frac{\hbar \delta E_{\mathrm{xc}}^{\mathrm{LDA}}}{2 \mu_{\mathrm{B}} \delta \vec{s}}\right|_{\substack{n(\vec{r}, t) \\ \vec{r}, t)}}
$$

from the instantaneous electron charge $n(\vec{r}, t)=\sum_{j \in \text { occ. }}$ $\psi_{j}^{*}(\vec{r}, t) \psi_{j}(\vec{r}, t) \quad$ and $\quad$ spin density $\quad \vec{s}(\vec{r}, t)=\frac{\hbar}{2} \sum_{j \in \text { occ. }}$ $\psi_{j}^{*}(\vec{r}, t) \vec{S} \psi_{j}(\vec{r}, t)$. For the XC magnetic field the zero-torque theorem holds [19], i.e., $\int d^{3} r \vec{B}_{\mathrm{xc}}(\vec{r}) \times \vec{s}(\vec{r})=0$, where the integral is taken over the entire space. In other words, $\vec{B}_{\mathrm{xc}}$ cannot produce a global spin torque over the system. The Heisenberg equation of motion for the spin operator leads to a spin continuity equation in the form

$\frac{d \vec{s}(\vec{r}, t)}{d t}=-\nabla \cdot \mathrm{J}_{\mathrm{KS}}(\vec{r}, t)+2 \frac{\mu_{\mathrm{B}}}{\hbar} \vec{s}(\vec{r}, t) \times \vec{B}_{\mathrm{xc}}(\vec{r}, t)+\vec{\Gamma}_{\mathrm{SO}}(\vec{r}, t)$

where

$$
\begin{aligned}
\vec{\Gamma}_{\mathrm{SO}}(\vec{r}, t)= & \sum_{\vec{R}_{I}} \sum_{\alpha, \beta} \sum_{m, m^{\prime}, l} \epsilon_{n j k} V_{l}^{\mathrm{SO}}\left(\left|\vec{r}-\vec{R}_{I}\right|\right) \\
& \cdot\left\langle\Psi_{\alpha}^{\mathrm{KS}} \mid l, m^{\prime}, \vec{R}_{I}\right\rangle \hat{S}_{\alpha, \beta}^{k} \\
& \cdot\left\langle l, m^{\prime}, \vec{R}_{I}\left|\hat{L}_{I}^{j}\right| l, m, \vec{R}_{I}\right\rangle\left\langle l, m, \vec{R}_{I} \mid \Psi_{\beta}^{\mathrm{KS}}\right\rangle .
\end{aligned}
$$

In the first term in Eq. (6), the expectation value of the KS spin-current operator $\mathrm{J}_{\mathrm{KS}}^{i j}(\vec{r}, t)=$ $\operatorname{Tr}\left[\hat{S}^{i} \sum_{k}\left(\psi_{k}^{*} \partial_{j} \psi_{k}-\psi_{k} \partial_{j} \psi_{k}^{*}\right)\right]$ is a rank-two tensor. The second term is the torque exerted locally by $\vec{B}_{\mathrm{xc}}(\vec{r}, t)$, which vanishes within the adiabatic LSDA $\left[\vec{B}_{\mathrm{xc}}^{A L D A}(\vec{r}) \| \vec{s}(\vec{r})\right]$. The first term, however, can be rewritten in a form $\frac{2 \mu_{\mathrm{B}}}{\hbar} \vec{s} \times \vec{B}_{\text {kin }}$, where the kinetic magnetic field [20] is defined as $\vec{B}_{\text {kin }}=\frac{1}{2 e n} \sum_{k} \nabla_{k}\left(n \nabla_{k} \vec{s}\right)$. An effective local magnetic field can thus be defined as $\vec{B}_{\text {eff }}(\vec{r}, t)=\vec{B}_{\text {kin }}(\vec{r}, t)+\vec{B}_{\mathrm{xc}}(\vec{r}, t)$. This

\footnotetext{
${ }^{1}$ Our fully-relativistic norm-conserving pseudopotentials have been generated with the multi-reference pseudopotential method as implemented in the APE code [18]. This includes, in addition to valence states, also the semicore ones ( $3 s$ and $3 p$ ), since semicore states play an important role in describing the chemical bond and the magnetic properties of transition metal clusters [22].

${ }^{2}$ For values of the radius greater than the range of the SOC component the ionic pseudopotential reduces to a purely local object.
} 

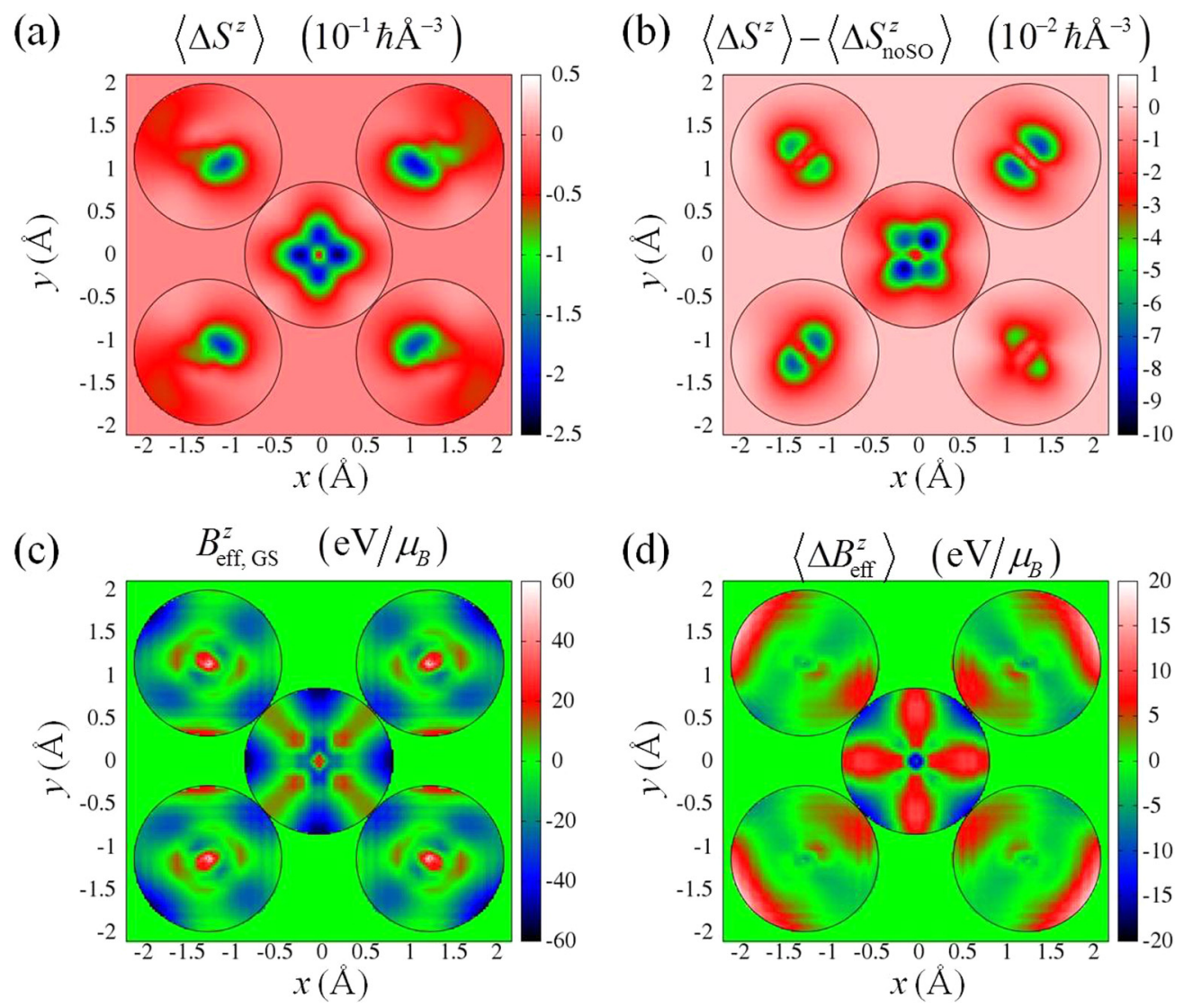

FIG. 2. Contour plots of the time-averaged and $z$-averaged observables evaluated only within spheres of radius $0.85 \AA$ around each atom: (a) variation of the spin density $S^{z}(\vec{r})$ with respect to the ground state, (b) difference of the latter and its counterpart in the case of no SOC, (c) the effective magnetic field $B_{\text {eff }}^{z}(\vec{r})$ in the ground state $(t=0)$, and (d) the variation of $B_{\text {eff }}^{z}(t)$ with respect to the ground state. See text for details.

field is not necessary locally parallel to $\vec{s}(\vec{r}, t)$ and produces a local torque.

The last term in Eq. (6) $\vec{\Gamma}_{\mathrm{SO}}(\vec{r}, t)$ is the only source of global spin dissipation in the temporal evolution of the spin density as a result of the SOC. Note that in this description the global spin change is only determined by the orbital-dependent scattering properties of the atomic pseudopotential as a result of the SOC. It is, therefore, not directly dependent on the KS orbital momentum distribution defined with respect to the center of mass of the cluster at $(0,0,0)$ as

$$
\vec{L}_{\mathrm{KS}}(\vec{r}, t)=\vec{r} \times \vec{j}_{\mathrm{KS}}(\vec{r}, t),
$$

where $\vec{j}_{\mathrm{KS}}=\hbar \sum_{k}\left(\psi_{k}^{*} \nabla \psi_{k}-\psi_{k} \nabla \psi_{k}^{*}\right) / 2 i$ is the KS probability current. Later in this paper we will revisit Eq. (6) and use it as a base for a simplified quantum model for the spin operator.

\section{DEMAGNETIZING $\mathrm{Fe}_{6}$ FROM TDSDFT}

Figure 1 shows representative results from the full timedependent simulations of the dynamics of the $\mathrm{Fe}_{6}$ cluster. For a range of pulse shapes and amplitudes the magnetic response is a decay in the global spin expectation value, $S_{\text {tot }}^{z}(t)=$ $\int d^{3} r s^{z}(\vec{r}, t)$. Pulses (with fluences comparable to those used in pump-probe experiments) excite the cluster, and the rate of the spin decay triggered by the excitation typically correlates with the total variation of the TDSDFT energy before and after the pulse (the larger the energy deposited in the cluster, the larger the induced spin-decay rate). Note that after the pulse the total energy is conserved, Fig. 1(b). The total orbital momentum, Fig. 1(c), defined as $L_{\text {tot }}^{z}(t)=\int d^{3} r L_{\mathrm{KS}}^{z}(\vec{r}, t)$, shows large oscillations and a very different behavior from that of the spin. We note that the total angular momentum in our description is not a constant of motion as the orbital momentum does not commute with the noncentral ionic potential of the cluster. In other words, the frozen nuclei constitute a sink for the orbital momentum (see Appendix). The global nonconservation of orbital momentum leads to global nonconservation of spin through the $\Gamma_{\text {So }}$ torque in Eq. (6).

The spatial distribution of the calculated demagnetization is visualized in Fig. 2, where we plot the time and space averaged (along the direction of the symmetry axis of the cluster, $z$ ) planar distributions of the temporal variations (with respect to the GS) of the spin-density and the effective magnetic field. ${ }^{3}$ It is notable that the negative variation of $s^{z}(\vec{r})$ is

\footnotetext{
${ }^{3}$ The definitions of the quantities plotted in Fig. 2 are: $\overline{\Delta S^{z}(x, y)}=$ $\frac{1}{T} \sum_{i} \int_{T} d t \int_{z_{i, 1}}^{z_{i, 2}} d z \quad\left[s^{z}(x, y, z, t)-s^{z}(x, y, z, 0)\right] /\left(z_{i, 2}-z_{i, 1}\right) \quad$ and $\overline{\Delta B_{\mathrm{eff}}^{z}(x, y)}=\frac{1}{T} \sum_{i} \int_{T} d t \int_{z_{i, 1}}^{z_{i, 2}} d z \quad\left[B_{\mathrm{eff}}^{z}(x, y, z, t)-B_{\mathrm{eff}}^{z}(x, y, z, 0)\right] /$ $\left(z_{i, 2}-z_{i, 1}\right)$, where $T$ is the total simulation time and $z_{i, 1}, z_{i, 2}$ are functions of $(x, y)$ and belong to one of the nonoverlapping atom-centered spheres $\Xi_{i}$.
} 

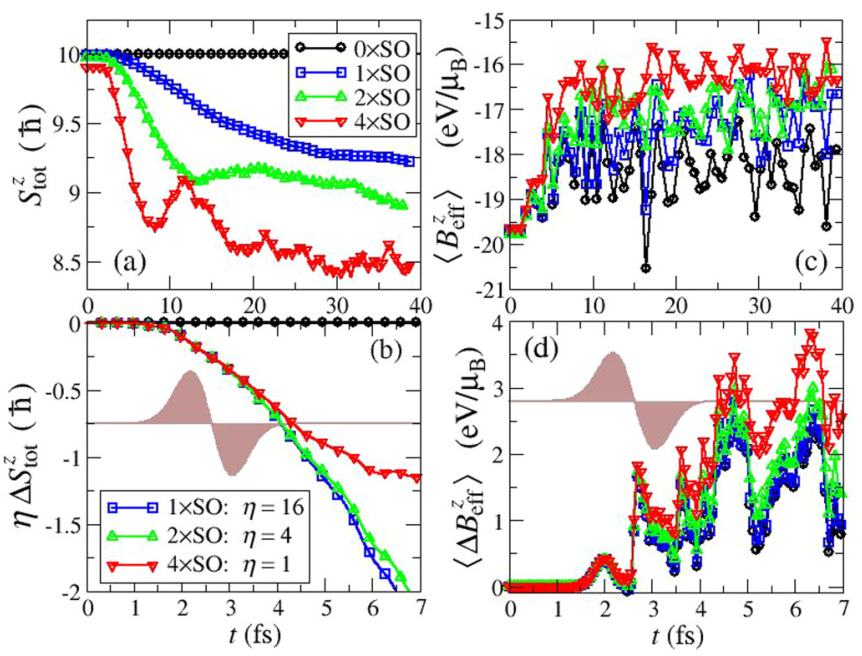

FIG. 3. (a) Evolution of global spin expectation value for different values of $\alpha=0,1,2,4$ in Eq. (4). (b) Same as in panel (a) but having the GS spin subtracted and $\Delta S_{\text {tot }}^{z}$ values multiplied by $\eta=\left(\alpha_{\max } / \alpha\right)^{2}$. Panels (c) and (d) show the corresponding trajectories of the averaged over the nonoverlapping atom-centered spheres $B_{\text {eff }}^{z}(t)$ [see Eq (9)].

predominantly localized around the atomic centers [panel (a)]. Furthermore, the difference in the averaged spin-variation distribution between analogous simulations with and without SOC [panel (b)], which approximately represents the global spin loss in the presence of the SOC, is also localized. ${ }^{4}$ In particular it is more pronounced along the direction of the bond with the apex atoms (which is also the shortest bond length in the system in the direction of the laser field). The effective magnetic field is very inhomogeneous in the GS. Its temporal variation, however, shows a spatial correlation with the variation of the spin density. The regions of decrease of spin density exhibit an increase in $B_{\text {eff }}^{z}(\vec{r})$ (note that in the same regions $B_{\mathrm{eff}}^{z}(\vec{r})$ is mostly negative in the GS). Hence, regions of pronounced spin decay are associated with decrease in the absolute value of $B_{\text {eff }}^{z}(\vec{r})$.

As suggested by Eq. (6), the SOC is expected to have a major role in the spin-decay process. In order to extract such effect we have introduced an artificial scaling factor $\alpha$ in front of the SOC term of Eq. (4). Depicted in Fig. 3 is the effect on the global spin-variation trajectory of the variation of $\alpha$ from 0 to 4 . The rate of spin loss, both pulse-coherent and post-pulse, is strongly affected by the SOC strength with the limit of $\alpha=0$ (no SOC) resulting in global spin conservation. In panel (b) we have plotted the same spin trajectories after removing their GS offset and scaling them by a factor of $1 / \alpha^{2}$. The overlap of the curves demonstrates that in the initial coherent stage the spin-decay rate scales as the square of the SOC strength.

We now compare the global spin trajectories to those of the $B_{\text {eff }}^{z}$ in the vicinity of the atomic centers. We define a measure of the local variation of the latter as

$$
\left\langle B_{\mathrm{eff}}^{z}(t)\right\rangle=\sum_{i} \frac{1}{V_{\Xi_{i}}} \int_{\Xi_{i}} B_{\mathrm{eff}}^{z}(\vec{r}, t) d^{3} r
$$

\footnotetext{
${ }^{4}$ The apparent spatial symmetry breaking is due to the finiteness of the time domain simulation.
}

where $\Xi_{i}$ are nonoverlapping atom-centered spheres of radius $0.85 \AA$ for all the quantities plotted in Figs. 3(c) and 3(d). Although such defined $\left\langle B_{\text {eff }}^{z}(t)\right\rangle$ appears noisy due to spatial grid effects, it does show a coherent response to the external field pulse and during this stage it is practically independent of the SOC strength. ${ }^{5}$ After the pulse dies out the decrease in the absolute value of $\left\langle B_{\text {eff }}^{z}(t)\right\rangle$ correlates to the global spin decay in panel (a), and that is especially notable for higher $\alpha$. This is related to the fact that practically all the spin loss takes place in the same atomic vicinity regions where $\left\langle B_{\mathrm{eff}}^{z}(t)\right\rangle$ is defined.

\section{MINIMAL SPIN MODEL FOR DEMAGNETIZATION}

The insights drawn from the first-principles spin dynamics results for $\mathrm{Fe}_{6}$ presented in Fig. 3 suggest that the $\mathrm{SOC}$ is key in the demagnetization process, which in turn takes place in the vicinity of the atomic sites, i.e., where the SOC is the strongest. Furthermore, we have observed that in the same regions the local magnetic field $\left\langle B_{\text {eff }}^{z}(t)\right\rangle$ also decays rapidly in time, coherently with the laser field in the beginning of the simulation. As a minimal model for understanding the demagnetization process we propose the following spatiallyhomogeneous and time-dependent spin Hamiltonian

$$
\hat{H}(t)=\lambda \hat{L} \cdot \hat{S}+\vec{B}(t) \cdot \hat{S},
$$

where $\lambda$ defines the SOC strength and $\vec{B}(t)$ is a time-dependent magnetic field. This model effectively mimics the local spin dynamics at a given point in space resulting from the TDSDFT description formulated in Eq. (6). The basis set used to expand the wave function, solution of the corresponding timedependent Schrödinger equation, is given by the eigenstates of $\hat{L}^{z}$ and $\hat{S}^{z},\left\{\left|l^{z}, s^{z}\right\rangle\right\}$. For instance, considering $l=1$ for the orbital quantum number we can write

$$
|\Psi(t)\rangle=\sum_{l^{z}=-1}^{1} \sum_{s^{z}=-1 / 2}^{1 / 2} c_{l^{z}, s^{z}}\left|l^{z}, s^{z}\right\rangle,
$$

and solve numerically the six-dimensional Schrödinger equation to obtain the evolution of the spin observables in time. Such numerical integration in the case of an absence of SOC $(\lambda=0)$ returns, as can be expected, no observable spin dynamics $\left(S^{z}=\right.$ const $)$ regardless of whether or not the initial state is collinear to $\vec{B}(t)$. This is because the spin and orbital angular momenta in this case are decoupled.

In contrast when $\lambda \neq 0$, an initial state with $l^{z} \neq \pm l$ and a steplike variation of $\vec{B}(t)$ [similarly to $\left\langle B_{\text {eff }}^{z}(t)\right\rangle$ in Fig. 3] produce a sharp change in the expectation value of $\hat{S}^{z}$ (see Fig. 4). In particular, for an initial spin-up state $\left(S^{z}=1 / 2\right)$ we find a decrease of $S^{z}$ after the drop of the magnetic field, while an initially down spin-state shows an increase in $S^{z}$. In other words, a change of the local magnetic field, combined with the SOC, leads to a decrease in the modulus of the expectation value of the spin. This remains valid even if initially the spin state is collinear with the field (assumed along the quantization axis) as long as $l^{z} \neq \pm l$ (noncollinear

\footnotetext{
${ }^{5} \mathrm{SOC}$ is a small energy scale compared to the deposited by the pulse kinetic energies (Fig. 1), and the kinetic magnetic field variation is mostly due to charge motion.
} 


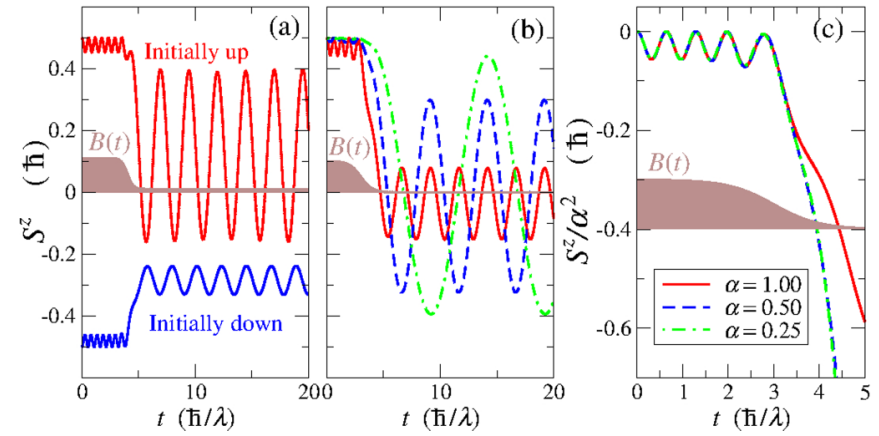

FIG. 4. Trajectories for $S^{z}$ corresponding to the model Hamiltonian of Eq. (10) in the case of (a) having an initial state with spin up or spin down or (b) different values of the SOC by a factor $\alpha$, such that $\lambda \rightarrow \alpha \lambda$ in Eq. (10), for an initial spin up. (c) Similarly to Fig. 2(b), the latter trajectories rescaled by $1 / \alpha^{2}$. The shaded area is a reference for the temporal profile of $\vec{B}=[0,0, B(t)]$. The initial orbital momentum state is a linear combination of $l^{z}=0,1,2$ states.

orbital momentum). ${ }^{6}$ Interestingly, this massively simplified local spin model reproduces the $\lambda^{2}$ dependence of the coherent demagnetization rate observed in the TDSDFT calculations [Fig. 3(b)].
In Fig. 4(b) we show the results of the numerical integration of our six-dimensional spin-orbital model for different values of the SOC strength, rescaled by a factor $\alpha$. It is demonstrated in panel (c) that the demagnetization curves during the variation of the magnetic pulse become steeper by a factor $\alpha^{2}$ as the rescaled trajectories $S^{z} / \alpha^{2}$ coincide for some time interval. This property can also be demonstrated analytically by deriving the lowest order terms in the perturbative expansion of the solution $|\Psi(t)\rangle$ in powers of $t$. For simplicity we consider the dynamics of the two coupled states $\left|l^{z}=1, s^{z}=-1 / 2\right\rangle$ and $\left|l^{z}=0, s^{z}=1 / 2\right\rangle$

$$
|\Psi(0)\rangle=c_{1}|1,-1 / 2\rangle+c_{2}|0,1 / 2\rangle,
$$

under the time-dependent Hamiltonian $\hat{H}(t)$ which has a matrix form

$$
H(t)=H_{0}+V(t)=\left(\begin{array}{cc}
-\frac{\lambda}{2} & \frac{\lambda}{\sqrt{2}} \\
\frac{\lambda}{\sqrt{2}} & 0
\end{array}\right)+\left(\begin{array}{cc}
-\frac{B(t)}{2} & 0 \\
0 & \frac{B(t)}{2}
\end{array}\right)
$$

where we have decomposed $\hat{H}(t)$ into a time-independent $\hat{H}_{0}$ and a time-dependent part $\hat{V}(t)$. In the interaction picture the operator $\hat{V}(t)$ and the spin $\hat{S}^{z}$ operator are represented as

$$
\begin{aligned}
& V_{I}(t)=e^{i H_{0} t} V(t) e^{-i H_{0} t}=\frac{1}{9}\left(\begin{array}{cc}
-\frac{B(t)}{2}-4 B(t) \cos \left(\frac{3}{2} \lambda t\right) & \sqrt{2} B(t)\left(1+e^{\frac{3}{2} i \lambda t}\right)-2 \sqrt{2} B(t) e^{-\frac{3}{2} i \lambda t} \\
\sqrt{2} B(t)\left(1+e^{-\frac{3}{2} i \lambda t}\right)-2 \sqrt{2} B(t) e^{\frac{3}{2} i \lambda t} & \frac{B(t)}{2}+4 B(t) \cos \left(\frac{3}{2} \lambda t\right)
\end{array}\right), \\
& S^{z}(t)=\frac{\hbar}{2} e^{i H_{0} t} \sigma^{z} e^{-i H_{0} t}=\frac{\hbar}{18}\left(\begin{array}{cc}
1+8 \cos \left(\frac{3}{2} \lambda t\right) & -2 \sqrt{2}+4 \sqrt{2} e^{-\frac{3}{2} i \lambda t}-2 \sqrt{2} e^{\frac{3}{2} i \lambda t} \\
-2 \sqrt{2}+4 \sqrt{2} e^{\frac{3}{2} i \lambda t}-2 \sqrt{2} e^{-\frac{3}{2} i \lambda t} & -1-8 \cos \left(\frac{3}{2} \lambda t\right)
\end{array}\right),
\end{aligned}
$$

where $\sigma^{z}$ is the corresponding Pauli matrix.

In the limit of $t \rightarrow 0$, the equation for the time-dependent wave function in the interaction picture

$$
\left|\Psi_{I}(t)\right\rangle=\left|\Psi_{0}\right\rangle-\frac{i}{\hbar} \int_{0}^{t} d t^{\prime} V_{I}\left(t^{\prime}\right)\left|\Psi_{I}\left(t^{\prime}\right)\right\rangle
$$

can be expanded in powers of $t$ as

$$
\left|\Psi_{I}(t)\right\rangle=\left|\Psi_{0}\right\rangle-\left.\frac{i}{2 \hbar} \frac{d}{d t} V_{I}(t)\right|_{t=0} t^{2}\left|\Psi_{0}\right\rangle+O\left(t^{3}\right)
$$

Restricting ourselves with terms of the order of $t^{2}$, the variation of expectation value of $\hat{S}^{z}$ between $\left|\Psi_{I}(t)\right\rangle$ and the ground state $\left|\Psi_{0}\right\rangle$ then reads

$$
\left\langle\hat{S}^{z}(t)\right\rangle-\left\langle\hat{S}^{z}\right\rangle_{0}=\frac{\lambda^{2} t^{2}}{2 \hbar}\left(-\frac{\sqrt{2}}{2} c_{1} c_{2}-c_{1}^{2}+c_{2}^{2}\right),
$$

i.e., it scales predominantly as $\lambda^{2}$ for small times $t$ following the initiation of the $B$-field variation. Note that in this approximation the early demagnetization does not depend on the strength of the field.

\footnotetext{
${ }^{6}$ Note that this requirement is not a particular restriction for the model. For instance, the valence states in the open $d$ shell of the magnetic transition-metal atoms satisfy it.
}

\section{MATERIAL-SPECIFIC DEMAGNETIZATION OF SIMILAR CLUSTERS}

As a final quantitative verification of the $\lambda^{2}$ dependence of the demagnetization speed, we look at the laser-induced response of clusters completely identical to $\mathrm{Fe}_{6}$ in terms of geometry but composed of $\mathrm{Co}$ and $\mathrm{Ni}$ (note that we keep the same frozen geometry of $\mathrm{Fe}_{6}$ as in Ref. [21], although this is not the GS for $\mathrm{Ni}_{6}$ and $\mathrm{Co}_{6}$, in order to exclude the structural factor from the comparison). We quantify their ionic SOC strength through the following definition

$$
\lambda_{\text {eff }}=\sum_{l \in \text { occ. }} \frac{n_{l}}{n_{\text {tot }}} \int V_{l}^{\mathrm{SO}_{(}(\vec{r}) R_{l}^{2}(\vec{r}) d^{3} r}
$$

where $n_{l}$ are the KS state occupations with $l$ spanning the valence states (in this case $3 s, 3 p, 3 d$ and $4 s$ ), $n_{\text {tot }}=$ $\sum_{l \in \text { occ. }} n_{l}, R_{l}(\vec{r})$ are the radial pseudoatomic wave functions and $V_{l}^{\mathrm{SO}}(\vec{r})=\frac{2 l}{2 l+1}\left[V_{\mathrm{PP}}^{l+1 / 2}(\vec{r})-V_{\mathrm{PP}}^{l-1 / 2}(\vec{r})\right]$ is the same object as in Eq. (4). Those are all obtained from LSDA calculations of isolated atoms. By fitting the first few femtoseconds of the demagnetization curve to a quadratic time decay $S^{z}(t) \propto$ $A\left(t-t_{0}\right)^{2}$ with $t_{0}$ in the rise of the laser pulse (around $1.5 \mathrm{fs}$ ) we extract the demagnetization rate $A$ of each cluster. Such extracted demagnetization rates for the three different clusters 

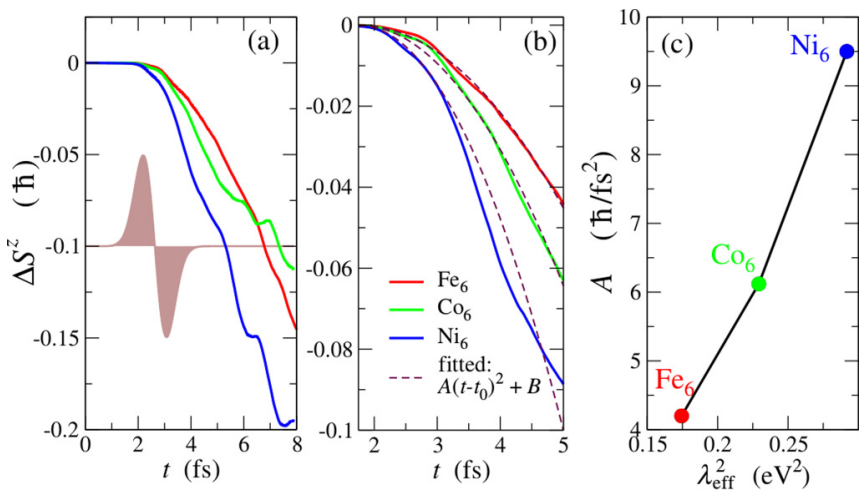

FIG. 5. (a) TDSDFT trajectories of the variation of $S_{\text {tot }}^{z}(t)$ with respect to the GS spin for three different clusters: $\mathrm{Fe}_{6}, \mathrm{Ni}_{6}$, and $\mathrm{Co}_{6}$, all sharing the same $\mathrm{Fe}_{6}$ geometry [12]. (b) Closer view into the coherent part of the trajectories where a parabolic decay $\left[y=A\left(t-t_{0}\right)^{2}+A_{0}\right]$ is fitted for each trajectory (dashed curves). (c) The fitting coefficients $A$ from panel (b) versus the effective atomic SOC from Eq. (16) for each material.

show a systematic dependence on their ionic SOC strength $\lambda_{\text {eff }}^{2}$ [see Fig. 5(c)].

\section{CONCLUSION}

In conclusion, TDSDFT calculations at the level of ALSDA, combined with fully relativistic pseudopotentials, display ultrafast demagnetization effect for a range of laser-excited transition-metal clusters with fixed geometry. The global demagnetization effect scales quadratically with time in the first instants, with rates ranging between 4 and $10 \hbar / \mathrm{fs}^{2}$, and strongly depends on the SOC strength. The phenomenon is then explained in terms of the resulting laser-induced coherent drop of the effective magnetic field. The latter, combined with the SOC which is the strongest in the vicinity of the atomic centers, leads to a local decrease of the expectation value of $\hat{S}^{z}$. The external electric pulse is therefore only indirectly involved in the demagnetization process, which could instead be directly ascribed to the large variations of the effective magnetic field due to excited spin-polarized currents. Furthermore, the onset demagnetization rate shows a strong dependence on the ionic SOC properties of the material, scaling quadratically with the SOC strength. Because of the localized nature of this ultrafast demagnetization mechanism, we believe our findings are valid beyond the cluster systems and may provide a formal backing to experimental observations like the comparison of $\mathrm{Ni}$ and $\mathrm{CoPt}_{3}$ demagnetization rates in Ref. [6].

\section{ACKNOWLEDGMENTS}

This work has been funded by the European Commission FP7 project CRONOS (Grant No. 280879) and by Science Foundation Ireland (Grant No. 14/IA/2624). We gratefully acknowledge the DJEI/DES/SFI/HEA Irish Centre for HighEnd Computing (ICHEC) for the provision of computational facilities and support and the Trinity Centre for High Performance Computing for technical support. M.S. is grateful to Tchavdar Todorov for a discussion on fundamental aspects of this work.

\section{APPENDIX}

In this Appendix we look in more detail into the problem of the nonconservation of the total angular momentum (TAM) in our time-dependent simulations. In fact, it might appear counterintuitive that the TAM of the electrons coupled to a system of frozen nuclei is not a constant of motion. Recently it has been demonstrated that the TAM of the quantum system made of an atomic spin (localized) coupled to an elastic medium (phonons) is conserved [21]. One may then be tempted to attribute also the dissipation of the electronic TAM (which also has an orbital component) to the single mechanism of the interaction with a phonon bath. As this is not available here one may then conclude that the TAM must be conserved.

The total orbital momentum of the system of electrons, laser pulse and lattice,

$$
\vec{L}=\vec{L}_{\mathrm{el}}+\vec{L}_{\text {laser }}+\vec{L}_{\text {lattice }},
$$

is not an observable that is straightforward to evaluate from a microscopical theory and within our TDSDFT framework it is not a constant of motion.

The Noether theorem guarantees the conservation of $\vec{L}$ in the case of a global rotational symmetry of the interactions in the system. This is not the case for the electron subsystem in the cluster considered here. But even if our electron Hamiltonian was spherically symmetric (e.g., a single Fe atom), the orbital momentum in Eq. (A1) would still not be a constant of motion. As the laser field is treated classically within the dipole approximation, it is not evolved dynamically but it is characterized only by a time-dependent and homogeneous electric field with zero Poynting vector. Hence, during the laser pulse the orbital momentum of Eq. (A1) is not conserved. As we consider the frozen-nuclei approximation, $\vec{L}_{\text {lattice }}=0$ during the evolution (since their velocity is zero they do not contribute to the orbital momentum).

In the TDSDFT simulations the laser pulse and the atomic nuclei constitute external sources of torque on the electronic component of the orbital momentum $\vec{L}_{\mathrm{el}}$ which we define as the KS orbital momentum $\vec{L}_{\mathrm{el}}=\vec{L}_{\mathrm{KS}}$ from Eq. (8). The evolution of $\vec{L}_{\mathrm{KS}}$ obeys the following equation of motion

$$
\begin{aligned}
\frac{d}{d t} \vec{L}_{\mathrm{el}}= & -\frac{q}{2 c} \int d^{3} r \vec{A}(t) \times \vec{j}_{K S}(\vec{r}, t) \\
& +\mu_{B} \sum_{j} \int d^{3} r(\vec{r} \times \nabla) B_{x c}^{j}(\vec{r}, t) m^{j}(\vec{r}, t) \\
& -\int d^{3} r \vec{r} \times \nabla v_{K S}(\vec{r}, t) \rho(\vec{r}, t) \\
& -\int d^{3} r \vec{\Gamma}_{\mathrm{SO}}(\vec{r}, t),
\end{aligned}
$$

which can be derived from basic quantum-mechanical principles given the KS Hamiltonian in Eq. (2).

The first of the four terms on the right hand side describes the variation of orbital momentum due to the action of the external electric field. The second one is a torque due to the scattering of the electrons with the field of the localized magnetic moments, while the third one describes the effect due to the scattering of the electrons with the field of the 
nuclei. The last two terms can also be expressed in terms of the commutator between the orbital momentum operator $\vec{L}_{\mathrm{el}}$ and the ionic pseudopotential

$$
\hat{V}_{\mathrm{PP}}(\vec{r})=\sum_{\vec{R}_{I}} \sum_{l, m_{1}, m_{2}} V_{\mathrm{ion}}^{l}\left(\left|r-R_{I}\right|\right)\left|l, m_{1}, I\right\rangle\left\langle l, m_{2}, I\right| .
$$

At time $t=0$ the GS density matrix commutes with the ionic pseudopotential $\hat{V}_{\mathrm{PP}}(\vec{r})$, and the average GS orbital momentum can be obtained integrating Eq. (8). During and after the laser excitation the set of spherical harmonics $\{|l, m, I\rangle\}$, centered on each of the atoms $I$, are not eigenfunctions of the Hamiltonian and the ionic potential becomes a source of scattering for the orbital momentum. As a result of the excitation the center of mass of the electron system acquires a finite orbital momentum in the reference frame of the frozen nuclei. The TAM of the electrons in not conserved but must oscillate on some large timescale after the decay of the external excitation. As a classical analog for such nonconservation of the angular momentum one could think of the case of a satellite orbiting a pair of heavy stationary planets (mass points) - the TAM of the satellite on a closed "8"-shaped orbit passing through the center of gravity of the binary system is oscillating around zero (the TAM in each loop around one of the planets clearly points in the opposite direction).
The fourth term in Eq. (A2) is the variation due to the spin-orbit coupling effect, which is opposite and equal to $\vec{\Gamma}_{\text {SO }}$ from Eq. (7). In the special case of a potential with central symmetry, $\vec{B}_{\mathrm{xc}}(\vec{r})$ and $v_{\mathrm{KS}}(\vec{r})$ depend only on the distance from the center of symmetry of the system, hence $\vec{r} \times \nabla B_{\mathrm{xc}}(r)=0$ and $\vec{r} \times \nabla v_{\mathrm{KS}}(r)=0$. For the long-time dynamics after the laser pulse is extinguished, $\vec{A}(t)=0$, and the equation reduces to

$$
\frac{d}{d t} \vec{L}_{\mathrm{el}}=-\int d^{3} r \vec{\Gamma}_{\mathrm{SO}}(\vec{r}, t) .
$$

In this case the SOC is the only source of variation for the orbital momentum and the total angular momentum $\vec{L}_{\mathrm{el}}+\vec{S}$ is a constant of motion. For instance, we find no significant demagnetization in the case of a single $\mathrm{Fe}$ atom under the same excitation. Hence we conclude that the presence of the symmetry-breaking lattice is essential for the laser-driven decay of the spin and the mechanism goes through the generated effective (kinetic) magnetic field as described in Sec. IV.

In general, when the second and third term in Eq. (A2) are not zero for the excited cluster, because of the lack of central symmetry, they constitute a source/drain for the orbital momentum. As a result $\vec{L}_{\mathrm{el}}+\vec{S}$ is not conserved, as we have observed in our TDSDFT simulations, and for the ferromagnetic clusters $S^{z}$ initially decreases following the excitation.
[1] E. Beaurepaire, J.-C. Merle, A. Daunois, and J.-Y. Bigot, Phys. Rev. Lett. 76, 4250 (1996).

[2] A.Kirilyuk, A. V. Kimel, and T. Rasing, Rev. Mod. Phys. 82, 2731 (2010).

[3] B. Koopmans, G. Malinowski, F. D. Longa, D. Steiauf, M. Fähnle, T. Roth, M. Cinchetti, and M. Aeschlimann, Nat. Mater. 9, 259 (2010).

[4] Y. Hinschberger and P.-A. Hervieux, Phys. Lett. A 376, 813 (2012).

[5] M. Battiato, K. Carva, and P. M. Oppeneer, Phys. Rev. Lett. 105, 027203 (2010).

[6] J.-Y. Bigot, M. Vomir, and E. Beurepaire, Nat. Phys. 5, 515 (2009).

[7] G. P. Zhang and W. Hübner, Phys. Rev. Lett. 85, 3025 (2000).

[8] K. Krieger, J. K. Dewhurst, P. Elliott, S. Sharma, and E. K. U. Gross, J. Chem. Theory Comput. 11, 4870 (2015).

[9] E. K. U. Gross and W. Kohn, Adv. Quant. Chem. 21, 255 (1990).

[10] Z. Qian and G. Vignale, Phys. Rev. Lett. 88, 056404 (2002).

[11] O. Dieguez, M. M. G. Alemany, C. Rey, P. Ordejon, and L. J. Gallego, Phys. Rev. B 63, 205407 (2001).
[12] G. L. Gutsev and C. W. Beuschlicher, J. Phys. Chem. 107, 7013 (2003).

[13] M. A. L. Marques and E. K. U. Gross, A Primer in Density Functional Theory, Lecture Notes in Physics, Vol. 620 (SpringerVerlag, Berlin, 2003).

[14] J. P. Perdew and Y. Wang, Phys. Rev. B 45, 13244 (1992).

[15] M. A. L. Marques, A. Castro, G. F. Bertsch, and A. Rubio, Comp. Phys. Comm. 151, 60 (2003).

[16] C. L. Reis, J. M. Pacheco, and J. L. Martins, Phys. Rev. B 68, 155111 (2003).

[17] L. Kleinman, Phys. Rev. B 21, 2630 (1980).

[18] M. J. T. Oliveira and F. Nogueira, Comp. Phys. Comm. 178, 524 (2008).

[19] K. Capelle, G. Vignale, and B. L. Györffy, Phys. Rev. Lett. 87, 206403 (2001).

[20] M. I. Katsnelson and V. P. Antropov, Phys. Rev. B 67, 140406(R) (2003).

[21] D. A. Garanin and E. M. Chudnovsky, Phys. Rev. B 92, 024421 (2015).

[22] J. Zhu, X. W. Wang, and S. G. Louie, Phys. Rev. B 45, 8887 (1992). 